\title{
Recent advances in the pathogenesis and management of Raynaud's phenomenon and digital ulcers
}

DOI:

10.1097/BOR.0000000000000332

\section{Document Version}

Accepted author manuscript

Link to publication record in Manchester Research Explorer

\section{Citation for published version (APA):}

Herrick, A. L. (2016). Recent advances in the pathogenesis and management of Raynaud's phenomenon and digital ulcers. Current Opinion in Rheumatology, 28(6), 577-85. https://doi.org/10.1097/BOR.0000000000000332

\section{Published in:}

Current Opinion in Rheumatology

\section{Citing this paper}

Please note that where the full-text provided on Manchester Research Explorer is the Author Accepted Manuscript or Proof version this may differ from the final Published version. If citing, it is advised that you check and use the publisher's definitive version.

\section{General rights}

Copyright and moral rights for the publications made accessible in the Research Explorer are retained by the authors and/or other copyright owners and it is a condition of accessing publications that users recognise and abide by the legal requirements associated with these rights.

\section{Takedown policy}

If you believe that this document breaches copyright please refer to the University of Manchester's Takedown Procedures [http://man.ac.uk/04Y6Bo] or contact uml.scholarlycommunications@manchester.ac.uk providing relevant details, so we can investigate your claim.

\section{OPEN ACCESS}


RECENT ADVANCES IN THE PATHOGENESIS AND MANAGEMENT OF RAYNAUD'S PHENOMENON AND DIGITAL ULCERS

Ariane L Herrick, MD, Centre for Musculoskeletal Research, The University of Manchester, Salford Royal NHS Foundation Trust, Manchester Academic Health Science Centre, Manchester, M13 9PT, UK and NIHR Manchester Musculoskeletal Biomedical Research Unit, Central Manchester NHS Foundation Trust, Manchester Academic Health Science Centre, UK.

Corresponding author: Ariane Herrick, The University of Manchester, Salford Royal NHS Foundation Trust, Manchester Academic Health Science Centre, Manchester UK. Telephone: 01612755993. Email: ariane.herrick@manchester.ac.uk 
ABSTRACT

Purpose of review: Systemic sclerosis (SSc)-related digital vasculopathy can progress from severe Raynaud's phenomenon (RP) to digital ulceration, is a major cause of pain and disability, and impacts negatively on quality of life. Current treatments are often ineffective and poorly tolerated. This review summarises some of the progress which has been made in the last 12 to 18 months in terms of our understanding of disease process, measurement and treatment.

Recent findings: The most important findings include that we can now better predict which patients with SSc are most likely to develop digital ulcers. In terms of treatment, a multicentre trial showed that the phosphodiesterase inhibitor sildenafil confers some benefit in SSc-related digital ulceration. Topical therapies are being explored: iontophoresis of vasodilators increases local blood flow, and in an avian model, $\mathrm{VEGF}_{121}$ fibrin applied in a gel matrix improved wound healing.

Summary: Progress is being made. Advances in our understanding of SSc-related vasculopathy continue to lead to exploration of new treatment approaches. Clinical trials and observational studies are challenging, but are being facilitated by developments in outcome measures and improved infrastructures and networking, allowing trials in much larger numbers of patients than have previously been possible.

KEY WORDS: Systemic sclerosis, Raynaud's phenomenon, digital ulcers, treatment 


\section{INTRODUCTION}

Raynaud's phenomenon (RP) occurs in almost all patients with systemic sclerosis (SSc) [1] and can be very severe, often progressing to digital ulceration (in around 50\% of patients) (Figure 1) and occasionally to gangrene $\left[2,3^{* *}\right]$. This severity relates to the fact that in SSc, structural as well as functional vascular abnormalities occur, in contrast to primary (idiopathic) RP when the vascular abnormality is thought to be purely functional and entirely reversible, never progressing to irreversible tissue injury. Recent studies have highlighted the burden of associated disability of both $\mathrm{RP}\left[4^{*}\right]$ and digital ulceration $\left[3^{* *}, 5\right]$, and the lack of effective treatments (only $16 \%$ of 443 patients with RP responding to a survey reported that current medication was effective [4*]).

A number of challenges confront clinicians and scientists with an interest in SSc-related digital vasculopathy, specific questions being:

1. What mechanisms drive the digital vasculopathy and lead to structural as well as functional change?

2. Can we predict which patients progress to digital ulceration?

3. Can we measure disease process reliably (in terms of both RP and digital ulcers), to provide outcome measures to facilitate clinical trials?

4. Are we making progress in developing safe and effective treatments, for both severe RP and SScrelated digital ulceration?

In this review I shall outline developments over the last 12-18 months in answering these four questions, under the headings of pathophysiology, predictors of digital ulceration, measurement of disease process, and management. Under each heading I shall consider firstly RP and secondly digital ulceration, although this is a false distinction because both are part of the spectum of SSc-digital vasculopathy. 


\section{PATHOPHYSIOLOGY}

\section{Raynaud's phenomenon}

The pathophysiology of RP is complex and most likely involves an interplay between vascular factors, neural control mechanisms and intravascular factors [6]. Flavahan, in a recent review [ $\left.7^{* *}\right]$, describes thermoregulatory mechanisms as central to an understanding of RP, highlighting the role of arteriovenous anastomoses, and how local cooling leads to increased activity of alpha $2_{2}^{-}$ adrenoceptors, resulting in reduced blood flow. In patients with SSc, structural microvascular changes mean that upstream vasoconstriction can irretrievably compromise nutritional blood flow and lead to tissue injury $\left[7^{* *}, 8\right]$. The review also gives a framework for targetted therapeutic interventions $\left[7^{* *}\right]$.

\section{Digital ulceration}

A commonly held view is that fingertip ulceration is ischaemic, whereas ulceration over the extensor surfaces of fingers is 'traumatic'. To date there has been very little direct evidence to support this theory. However, a study by Ruaro et al [9*] of 20 patients with SSc and fingertip ulcers demonstrated that blood flow is reduced at the site of fingertip ulcers (and improves with healing).

Saigusa et al performed a series of experiments investigating the role of CCN1 (a secreted cysteinerich heparin-binding protein) in SSc, and reported reduced circulating levels in patients with current or previous digital ulcers [10*]: they postulated that the reduced levels were at least in part caused by Fli1 deficiency. The same investigators $\left[11^{*}\right]$ suggested that Fli1 deficiency might result also in downregulation of endothelial protein C receptor (EPCR), serum levels of which were reduced in patients with digital ulcers compared to those without: reduced levels of EPCR could lead to a prothrombotic state which might contribute to ulceration by futher compromising microcirulatory 
flow. Therefore work is ongoing examining the cellular and molecular mechanims underpinning the vascular abnormalities which drive digital vasculopathy.

A review article by Chora et al [12*] summarised reported correlations between a large number of 'vascular biomarkers' and digital ulcers, and also with nailfold capillaroscopic change. Although associations do not prove causation, nonetheless it is tempting to speculate that some of the associates of digital ulceration might in the future be targets for therapy.

\section{PREDICTORS OF DIGITAL ULCERATION}

Accurate prediction of which patients with SSc are most likely to develop digital ulcers would allow risk stratification, relevant not only to clinical practice but also in the design of future clinical trials aimed at ulcer prevention.

Several studies in the last 18 months have described predictors of digital ulceration $\left[13^{*}, 14^{*}, 15^{*}, 16,17^{*}\right]$, and Manfredi et al described a prediction risk chart [18]. A large prospective study 623 patients with SSc from 59 centres reported that of the 468 patients with a history of previous digital ulceration, the capillary density in the middle finger of the dominant hand, the number of digital ulcers, and the presence of critical ischaemia, at baseline, were the strongest risk factors for development of new digital ulcers over the following six months [15*], therefore lending further support to previous studies suggesting that abnormal capillaroscopy (including reduced capillary density) is associated with future development of digital ulcers $\left[13^{*}, 19,20\right]$. Other predictors from recent studies included anti-topoisomerase antibodies $\left[16,17^{*}\right]$, the presence of autoantibodies against endothelin 1 (ET-1) Type A receptor [14*] and increased circulating levels of ET-1 [13*] and (reflecting functional vascular change), impaired thermal hyperaemia [21], endothelial dysfunction as assessed by flow-mediated dilatation [13*] and the severity of change on thermography $\left[22^{*}\right]$. 
A recent systematic review $\left[23^{*}\right]$ summarised risk factors and concluded that the diffuse cutaneous disease subtype, early onset RP/disease, anti-topoisomerase antibodies, capillaroscopy pattern, raised ET-1 levels, and low vascular endothelial growth factor (VEGF) levels were all risk factors for digital ulcers.

How can the clinican synthesise all these findings? In everyday clinical practice, those patients most likely to ulcerate are those with a history of severe RP/previous ulcers, antibodies to topoisomerase, and severe change on capillaroscopy (this last makes sense: the more severe the microvascular disease, the higher the likelihood of ischaemic ulcers). Other markers may be useful in the research setting but require further validation. It is worth highlighting that digital ulceration in patients with SSc has recently been shown to be a predictor of internal involvement and reduced survival [24*, $\left.25^{* *}\right]$.

\section{MEASUREMENT OF DISEASE PROCESS}

Raynaud's phenomenon

The only validated measure of RP is the Raynaud's Condition Score [26]. Clinical trials tend to rely on this and other patient reported outcomes, namely frequency, duration and severity of attacks. However, patient reported outcome measures are subjective, and a vision is to develop objective, non-invasive measures of digital blood flow: although these are unlikely to be used in large multicentre studies they have potential in proof-of-concept, early phase studies. Methods currently being used include thermography (an indirect measure of blood flow) and laser Doppler techniques $\left[27,28,29 *, 30^{*}\right]$. However, full validation and standardisation of protocols is required before these can be used more widely.

\section{Digital ulceration}


Digital ulcers are often a primary endpoint in randomised controlled trials (RCTs), and yet there is poor agreement as to their definition $[31,32]$. A recent study [33*] showed that adding contextual information (e.g presence of pain, discharge) to the visible appearance of the ulcers did not improve reliability. International efforts to standardise definition are ongoing.

\section{TREATMENT}

The purpose of this article is not to provide a state-of-the-art review of management but rather to highlight key recent advances. The UK Scleroderma Study Group consensus best practice pathways [34*] provides flow-charts of current management of RP, digital ulceration and critical ischaemia, but with the proviso (as discussed below) that these pathways are already slightly outdated because phosphodiesterase type 5 (PDE5) inhibitors should now be 'moved up' the pathways. Clinical practice will vary between countries depending on accessibility to the different treatments. Other than bosentan, the drugs discussed are not licenced for SSc-related digital vasculopathy.

Raynaud's phenomenon (which has not progressed to digital ulceration or critical ischaemia)

A recent systematic review highlighted the lack of evidence base for treatment of SSc-related RP [35]. However, progress is being made. The major point to highlight in the last 18 months for the practising clinician has been the increased use of PDE5 inhibitors, and many clinicians are now very likely to use a PDE5 inhibitor as a second choice after a calcium channel blocker in patients with SScrelated RP. This is because (a) PDE5 inhibitors have now been shown to confer benefit in a number of randomised controlled trials [36,37,38,39], and in a recent meta-analysis [40] (although the trials were all short-term, with a treatment period of 6 weeks or less) and (b) reduced costs of PDE5 inhibition from previously. Figure 2 is a modification of the UK best practice pathway, with a repositioning of PDE5 inhibitors alongside other oral vasoactive therapies. A recent study from the German Network for Systemic Sclerosis registry showed PDE5 inhibitors were prescribed in only $1.5 \%$ of patients with SSc-related RP (but without digital ulcers) first registered after 2009 , and in 
$5.5 \%$ of those with digital ulcers [41*]. Likewise numbers of patients prescribed PDE5 inhibitors in the recent Digital Ulcer Outcomes Registry (DUO) study were very low [3**]. It will be interesting to see how the situation changes over the next two to three years.

As Figure 2 outlines, intravenous prostanoids are used in some cases of severe 'uncomplicated' RP (RP which has not progressed to digital ulceration or critical ischaemia), but require hospitalisation. Selexipag, an oral IP prostacyclin receptor agonist which has been shown to be effective in pulmonary arterial hypertension, held promise also for RP. Disappointingly a recent clinical trial, so far published only in abstract form [42], showed no benefit from selexipag in SSc-related RP.

It seems likely that in SSc-related digital vasculopathy as in pulmonary arterial hypertension, combination therapy may be helpful given that different classes of drugs have very differing (and potentially synergistic) modes of action. Bellando-Randone et al [43] reported a retrospective study of 123 patients with SSc and suggested that combination treatment with bosentan and sildenafil conferred benefit on RP. Prospective studies are required.

Randomised controlled trials of RP are, however, challenging, given the heterogeneity of RP, the requirement to run studies over the winter months, and the subjectivity of patient reported outcome measures. Early phase proof-of-concept studies allow early assessment of promising therapies. Bose et al [44*] reported a 12 week double-blind, parallel-group, controlled trial comparing the endothelin Type A receptor antagonist ambrisentan (15 patients) with placebo (5 patients). Although no improvement in finger blood flow was demonstrated with ambrisentan (as assessed by laser Doppler perfusion imaging), as the authors concluded, there are a number of different mechanisms through which ET-1 blockade could exert benefit in SSc-related digital vasculopathy and ET-1 receptor antagonists warrent further research.

\section{Digital ulcers}


Several recent reviews give detailed descriptions of management $\left[45^{*}, 46^{*}, 47^{*}\right]$, with Figure 3 providing a summary flow-chart of current practice. Recent studies have highlighted the importance of adequate analgesia[48,49]: this is welcome as this key aspect of care is sometimes overlooked.

Randomised controlled trials. Three large scale randomised placebo-controlled trials of SSc-related digital ulceration have been published in the last year. The SEDUCE study of 83 evaluable patients (with 192 digital ulcers), compared 12 weeks' treatment with sildenafil 20mg three times daily to placebo (1:1 randomisation)[50**]. Although the primary end-point (time to healing) was not reached, sildenafil conferred some benefit with a greater healing rate in the sildenafil group compared to placebo at week $8(p=0.01)$ and week $12(p=0.03)$. The DUAL-1 and DUAL-2 studies[51**], randomising 289 and 265 patients respectively, both compared (over 16 weeks) macitentan 3mg daily, macitentan 10mg daily and placebo (1:1:1 randomisation). Macitentan, similarly to bosentan (which is licenced for the prevention of SSc-related digital ulcers) is a dual ETreceptor antagonist but has sustained receptor binding and increased tissue penetration. It reduces morbidity and mortality in pulmonary arterial hypertension [52] and was therefore a promising treatment for SSc-related digital ulcers. However, the cumulative number of new ulcers (the primary endpoint for each of DUAL-1 and DUAL-2) was no different between macitentan and placebo treatment: DUAL-2 was halted prematurely on the recommendation of the independent data monitoring committee. Irrespective of their outcomes, the SEDUCE and DUAL studies are testimony to the ability of the international community to mount large scale multicentre studies of SSc-digital ulcers. This represents a major step forward, and will facilitate addressing the need (highlighted by a meta-analysis in 2013 [53]) for larger studies with standardised outcome measures.

Observational studies of vasoactive therapies. The RAPIDS-1 and RAPIDS- 2 randomised controlled trials studies demonstrated the efficacy of bosentan in the prevention of SSc-related digital ulceration $[54,55]$. Agard et al $\left[56^{*}\right]$ conducted a retrospective study in 10 French centres to examine characteristics of 89 patients treated with bosentan in a 'real-world' setting: patients 
treated wth bosentan (median treatment duration until data collection was 17.5 months) had severe disease (61\% with at least 2 previous digital ulcer episodes, and $63 \%$ had had previous intravenous iloprost infusions). As with 'uncomplicated' RP, there is increasing interest in combination therapy: a retrospective analysis of 34 patients suggested that adding bosentan to monthly iloprost infusions was associated with ulcer healing in around $50 \%$ of patients [57]. While ideally controlled trials of combination therapy are needed, study design will prove highly challenging.

Procedural treatments. In SSc, these tend to be confined to patients who have progressed to digital ulceration or critical ischaemia. The increasing interest in botulinum toxin injections continues [58], with a prospective study of 20 patients with SSc reporting improvement in hand function after 8 weeks [59]. Randomised controlled trials are required and are underway.

Digital sympathectomy $[60,61]$ also continues to attract interest and is probably being performed increasingly in specialist centres. A recent retrospective study of 17 patients with SSc (26 hands operated on) reported symptomatic improvement in pain in $92.3 \%$ of hands and ulcer healing in all patients [62*], with the authors suggesting that the procedure should be considered earlier on in the disease rather than as a 'last resort'.

Fat grafting has been proposed as another possible therapy for severe secondary RP [63].

Extracorporeal shock wave therapy was reported to confirm some benefit in a study of 9 patients with SSc and 49 ulcers [64].

Therefore more 'aggressive' approaches to digital ulceration are being investigated and gaining ground. Although a systematic review concluded that the evidence base for surgical procedures for $\mathrm{RP}$ is lacking $\left[65^{*}\right]$, this is perhaps unsurprising given the relatively small numbers of patients coming to surgery and the difficulties in mounting clinical trials. Observational studies with standardised outcome measures should be encouraged. 
Topical therapies. Many patients with RP, with or without digital ulcers, do not tolerate oral or intravenous treatment because of systemic vasodilatory side effects. Effective topical therapies which act locally on the digits, and which are free from systemic side effects, are badly needed. It is encouraging that both preclinical and human studies are exploring topical delivery systems, including with iontophoresis, to improve blood flow $[66,67,68]$. An exciting potential development is the prospect of being able to apply local therapies to ulcers to induce angiogenesis and promote healing: a study in UCD-206 chickens showed that application of VEGF 121 -fibrin in a gel matrix improved ischaemic comb and neck lesions, with increased microvascular density compared to in fibrin treated and untreated lesions $[69 * *]$.

\section{CONCLUSION}

Advances in the understanding and treatment of SSc-related digital vasculopathy have been published in the last 12-18 months. Patients at high risk of progressing to digital ulcers can now be better identified, paving the way for studies of early intervention. Large multicentre studies can and are being mounted, and also smaller proof of concept studies of novel treatment modalities (facilited by standardisation of outcome measures). The next five years should see further translation of research findings into patient benefit.

WORD COUNT: 2528 


\section{KEY POINTS}

1. Digital vasculopathy is one of the major contributors to pain and disability in patients with SSc.

2. Pathophysiology remains elusive, but more is now known about the mechanisms of thermoregulatory imbalance and of structural vasculopathy.

3. Research is ongoing into outcome measures, including into definition of digital ulcers.

4. Randomised controlled trials, both early phase and larger multicentre, are feasible and providing an evidence base to treatment (e.g. for PDE5 inhibitors).

5. Other treatment approaches (e.g. botulinum toxin injections) are being advocated, but controlled clinical trials and/or carefully designed observational studies are required. 


\section{REFERENCES}

1. Meier FM, Frommer KW, Dinser R, et al. Update on the profile of the EUSTAR cohort: an analysis of the EULAR Scleroderma Trials and Research group database. Ann Rheum Dis 2012;71: 1355-1360.

2. Nihtyanova SI, Brough GM, Black CM, Denton CP. Clinical burden of digital vasculopathy in limited and diffuse cutaneous systemic sclerosis. Ann Rheum Dis 2008; 67: 120-123.

3. **Matucci-Cerinic M, Kreig T, Guillevin L, et al. Eludicating the burden of recurrent and chronic digital ulcers in systemic sclerosis: long-term results from the DUO registry. Ann Rheum Dis 2015 (EPub ahead of press).

This large study of 1459 patients with SSc included 674 patients with recurrent digital ulcers, and 164 with 'chronic' ulcers, and benchmarked the burden of morbidity and disability (including work impairment) attributable to digital ulcers.

4. *Hughes M, Snapir A, Wilkinson J, et al. Prediction and impact of attacks of Raynaud's phenomenon, as judged by patient perception. Rheumatology 2015; 54: 1443-1447.

This survey (443 respondents) highlighted the burden of disability from both primary and secondary $\mathrm{RP}$ and the lack of effective treatments as judged by patient opinion.

5. Brand M, Hollaender R, Rosenberg D, et al. An observational cohort study of patients with newly diagnosed digital ulcer disease secondary to systemic sclerosis registered in the EUSTAR database. Clin Exp Rheumatol 2015; 33 (Suppl. 91): S47-S54.

6. Herrick AL. The pathogenesis, diagnosis and treatment of Raynaud phenomenon. Nat Rev Rheumatol 2012; 8: 469-479.

7. **Flavahan NA. A vascular mechanistic approach to understanding Raynaud phenomenon. Nat Rev Rheumatol 2015; 11: 146-158. 
This review comprehensively discusses the vascular mechanisms underpinning Raynaud's

phenomenon (and thermoregulation more generally), including the basis for colour change. Possible new approaches to therapy are discussed.

8. Flavahan NA. Pathophysiological regulation of the cutaneous vascular system. In: Raynaud's Phenomenon: A Guide to Pathogenesis and Treatment. Edited by FM Wigley, AL Herrick, NA Flavahan (editors). New York: Springer; 2015. pp57-79.

9. *Ruaro B, Sulli A, Smith V, et al. Short-term follow-up of digital ulcers by laser speckle contrast analysis in systemic sclerosis patients. Microvascular Res 2015; 101: 82-85.

In this study, reduced blood flow at the site of fingertip ulcers (and which increased with healing) was demonstrated using the non-invasive technique of laser speckle contrast analysis.

10. *Saigusa R, Asano Y, Taniguchi, et al. A possible contribution of endothelial CCN1 downregulation due to Fli1 deficiency to the development of digital ulcers in systemic sclerosis. Exp Dermatol 2015; 24: $127-132$.

The authors describe a series of experiments implicating endothelial CCN1 downregulation in the development of SSc-related digital ulcers.

11. *Saigusa R, Asano Y, Yamashiti T, et al. Fli1 deficiency contributes to the downregulation of endothelial protein $\mathrm{C}$ receptor in systemic sclerosis: a possible role in prothrombotic conditions. $\mathrm{Br} \mathrm{J}$ Dermatol 2016; 174: 338-347.

This paper draws attention to the role of impaired coagulation and fibrinolysis in the pathogenesis of SSc, with particular reference including to digital ulcers.

12. * Chora I, Guiducci S, Manetti M, et al. Vascular biomarkers and correlation with peripheral vasculopathy in systemic sclerosis. Autoimmunity Reviews 2015: 14: 314-322. 
This review article gives a comprehensive list of vascular biomarkers, and their associations with nailfold capillaroscopic change and/or digital ulcers, in addition to putting the different biomarkers into context.

13. *Silva I, Teixeira A, Oliveira J, et al. Endothelial dysfunction and nailfold videocapillaroscopy pattern as predictors of digital ulcers in systemic sclerosis: a cohort study and review of the literature. Clinic Rev Allerg Immunol 2015; 49: 240-252.

This prospective observational study followed 77 patients with SSc for 3 years and examined predictors of digital ulcers in those with $(n=38)$ and those without $(n=39)$ existing digital ulcers. 14. *Avouac J, Riemekasten G, Meune C, et al. Autoantibodies against endothelin 1 Type A receptor are strong predictors of digital ulcers in systemic sclerosis. J Rheumatol 2015; 42: 1801-1807. This prospective cohort study of 90 patients reported that over the 5-year follow-up, 24 patients developed at least one new ulcer: anti-endothelin 1 Type A receptor autoantibodies together with a history of digital ulcers at baseline predicted new lesions.

15. *Cutolo M, Herrick AL, Distler O, et al. Nailfold videocapillaroscopic and other clinical risk factors for digital ulcers in systemic sclerosis: a multicenter, prospective cohort study. Arthritis Rheumatol 2016 (Epub ahead of print).

This prospective study of 623 patients (59 centres from 14 countries) reported that among the 468 patients with a previous history of digital ulceration, the three strongest predictors of new ulcers over 6 months were (at baseline) the mean number of capillaries $/ \mathrm{mm}$ in the middle finger of the dominant hand, the number of digital ulcers, and the presence of critical ischaemia.

16. Hunzelmann N, Riemekasten G, Becker MO, et al. The Predict Study: low risk for digital ulcer development in patients with systemic sclerosis with increasing disease duration and lack of topoisomerase-1 antibodies. Br J Dermatol 2016 (Epub ahead of print). 
17. *Wirz EG, Jaeger VK, Allanore $\mathrm{Y}$, et al. Incidence and predictors of cutaneous manifestations during the early course of systemic sclerosis: a 10-year longitudinal study from the EUSTAR database. Ann Rheum Dis 2016 (Epub ahead of print).

The authors report 10 year outcome in 695 patients with SSc from the European Scleroderma Trials and Research (EUSTAR) cohort who were seen within one year of onset of RP: on multivariate analysis, anti-topoisomerase antibodies were associated with development of digital ulcers.

18. Manfredi A, Sebastiani M, Carraro V, et al. Prediction risk chart for scleroderma digital ulcers: a composite predictive model based on capillaroscopic, demographic and clinico-serological parameters. Clin Hemorheol Microcirc 2015;59: 133-143.

19. Sebastiani M, Manfredi A, Colaci M, et al. Capillaroscopic skin ulcer risk index: a new prognostic tool for digital skin ulcer development in systemic sclerosis patients. Arthritis Rheum 2009; 61: 688694.

20. Smith V, De Keyser F, Pizzorni C, et al. Nailfold capillaroscopy for day-to-day clinical use: construction of a simple scoring modality as a clinical prognostic index for digital trophic lesions. Ann Rheum Dis 2011;70: 180-183.

21. Blaise $S$, Roustit $M$, Carpentier $P$, et al. The digital thermal hyperemia pattern is associated with the onset of digital ulcerations in systemic sclerosis during 3 years of follow-up. Microvascular Research 2014; 94: 119-122.

22. *Hughes M, Wilkinson J, Moore T et al. Thermographic abnormalities are associated with future digital ulcers and death in patients with systemic sclerosis. J Rheumatol (in press).

This retrospective review of 138 patients with SSc reported that those with abnormal thermography (defined as a temperature gradient between one or more of the fingertips and the dorsum of the 
hand of $>1^{\circ} \mathrm{C}$ at $30^{\circ} \mathrm{C}$ ) were more likely to develop digital ulcers than those with normal thermography.

23. *Silva I, Almeida J, Vasoncelos C. A PRISMA-driven systematic review for predictive risk factors of digital ulcers in systemic sclerosis patients. Autoimmunity Reviews 2015; 14: 140-152.

This is a comprehensive review of risk factors for SSc-related digital ulcers.

24. *Bruni C, Guiducci S, Bellando-Randone S, et al. Digital ulcers as a sentinel sign for early internal organ involvement in very early systemic sclerosis. Rheumatology 2015;54:72-76.

This study of 110 patients with a very early diagnosis of SSc (VEDOSS) included 25 with digital ulcers, all of whom had pulmonary and/or gastro-intestinal involvement of their disease: the conclusion was that digital ulcers were a 'sentinel sign' for internal organ invoelvement.

25. ** Mihai C, Landewe R, van der Heijde D et al. Digital ulcers predict a worse disease course in patients with systemic sclerosis. Ann Rheum Dis 2016; 75: 681-686.

1092 of 3196 patients (34.1\%) included into this study (from the EULAR Scleroderma Trials and Research [EUSTAR] database) had a history of digital ulcers at presentation: these patients were more likely to develop cardiovascular worsening or to die during the follow up of $5.0+2.2$ years than those without a history of ulcers.

26. Merkel PA, Herlyn K, Martin RW, et al. Measuring disease activity and functional status in patients with scleroderma and Raynaud's phenomemon. Arthritis Rheum 2002; 46: 2410-2420.

27. Dinsdale G, Herrick AL. Vascular diagnostics for Raynaud's phenomenon. J Vascular Diagnostics 2014; 2: 127-139.

28. Herrick AL, Murray AK, Ruck A, et al. A double-blind randomised placebo-controlled crossover trial of the alpha ${ }_{2 c}$-adrenoceptor antagonist ORM-12741 for prevention of cold-induced vasospasm in patients with systemic sclerosis. Rheumatology 2014; 53: 948-952. 
29. *Meijs J, Voskuyl AE, Bloemsaat-Minekus JP, Vonk MC. Blood flow in the hands of a predefined homogenous systemic sclerosis population: the presence of digital ulcers and the improvement with bosentan. Rheumatology 2015; 54: 262-269.

This study used laser Doppler perfusion imaging (LDPI) to evaluate treatment response.

30. *Pauling JD, Shipley JA, Hart DJ, et al. Use of laser speckle contrast imaging to assess digital microvascular function in primary Raynaud phenomeon and systemic sclerosis: a comparison using the Raynaud Condition Score Diary. J Rheumatol 2015; 42: 1163-1168.

A key finding of this cross-sectional study was poor correlation between subjective (Raynaud Condition Score) and objective (laser speckle contrast imaging and infrared thermography) assessments of Raynaud's phenomenon.

31. Herrick AL, Roberts C, Tracey A, et al. Lack of agreement between rheumatologists in defining digital ulceration in systemic sclerosis. Arthritis Rheum 2009; 60: 878-882.

32. Baron M, Chung L, Gyger G. Consensus opinion of a North American Working Group regarding the classification of digital ulcers in systemic sclerosis. Clin Rheumatol 2014; 33: 207-214.

33. *Hughes M, Roberts C, Tracey A, Dinsdale G, Murray A, Herrick AL. Does the clinical context improve the reliability of rheumatologists grading digital ulcers in systemic sclerosis? Arthritis Care Res (in press).

This study showed that the overall intra and inter-rater reliability of digital ulcer grading did not significantly improve with the clinical context and indicates the need for further research in defining digital ulcers.

34. *Hughes M, Ong VH, Anderson ME, et al. Consensus best practice pathway of the UK Scleroderma Study Group: digital vasculopathy in systemic sclerosis. Rheumatology 2015; 54: $2015-$ 2024. 
This consensus pathway provides a framework for clinicians regarding the different 'non-drug', drug and surgical treatments of RP and of SSc-related digital ulceration and critical digital ischaemia, including three flow-charts.

35. Garcia de la Pena Lefebvre P, Nishishinya MB, Pereda CA et al. Efficacy of Raynaud's phenomenon and digital ulcer pharmacological treatment in systemic sclerosis patients: a systematic literature review. Rheumatol Int 2015; 35: 1447-1459.

36. Shenoy PD, Kumar S, Jha LK, et al. Efficacy of tadalafil in secondary Raynaud's phenomenon resistant to vasodilator therapy: a double-blind randomized cross-over trial. Rheumatol 2010, 49: 2420-2428.

37. Herrick AL, Van den Hoogen F, Gabrielli A, et al. Modified-Release sildenafil reduces Raynaud's phenomenon attack frequency in limited cutaneous systemic sclerosis. Arthritis Rheum 2011, 63: 775-782.

38. Caglayan E, Axmann S, Hellmich M, et al. Vardenafil for the treatment of Raynaud phenomenon: a randomized, double-blind, placebo-controlled crossover study. Arch Intern Med 2012, 172: 11821184.

39. Lee EY, Park JK, Lee W, et al. Head-to-head comparison of udenafil vs amlodipine in the treatment of secondary Raynaud's phenomenon: a double-blind, randomized, cross-over study. Rheumatol 2014; 53: 658-664.

40. Roustit M, Blaise S, Allanore $\mathrm{Y}$, et al. Phosphodiesterase-5 inhibitors for the treatment of secondary Raynaud's phenomenon: systematic review and meta-analysis of randomised trials. Ann Rheum Dis 2013; 72: 1696-1699.

41. * Moinzadeh P, Riemekasten G, Siegert E, et al. Vasoactive therapy in systemic sclerosis: real-life therapeutic practice in more than 3000 patients. J Rheumatol 2016; 43: 66-74. 
The importance of this study is that it gives 'real-world' data about the use of different therapies (including for RP and digital ulceration) in patients with SSc (German Network for Systemic Sclerosis registry data), including comparison of before 2005 and after 2009.

42. Denton CP, Hachulla E, Riemekasten G, et al. Selexipag in Raynaud's phenomenon secondary to systemic sclerosis: a randomised, placebo-controlled, phase II study. Ann Rheum Dis 2016 (Abstract suppl).

43. Bellando-Randone $\mathrm{S}$, Lepri G, Bruni, $\mathrm{C}$ et al. Combination therapy with bosentan and sildenafil improves Raynaud's phenomenon and fosters the recovery of microvascular involvement in systemic sclerosis. Clin Rheumatol 2016; 35: 127-132.

44. *Bose N, Bena J, Chatterjee C. Evaluation of the effect of ambrisentan on digital microvascular flow in patients with systemic sclerosis using laser Doppler perfusion imaging: a 12-week randomized double-blind placebo controlled trial. Arthritis Res Therapy 2015: 17: 44.

This study highlights how non-invasive measures of blood flow can be used to quantify responsiveness to vasoactive treatments in the context of early phase studies, and how these measures may give different results from patient reported outcomes.

45. *Abraham S, Steen V. Optimal management of digital ulcers in systemic sclerosis. Therapeutics Clin Risk Management 2015; 11: 939-947.

This review gives the reader an up-to-date overview of the different aspects of management of SSrelated digital ulcers, including brief descriptions of the studies and reports underpinning the evidence base.

46. *Cappelli L, Wigley FM. Management of Raynaud phenomenon annd digital ulcers in scleroderma. Rheum Dis Clin N Am 2015; 41: 419-438. 
A highlight of this review is three case histories which give the reader a practical approach to management of different severities of SSc-related digital vasulopathy.

47. *Hughes M, Herrick AL. Digital ulcers in systemic sclerosis. Rheumatology (EPub ahead of print).

This review discusses management of SSc-related digital ulcers.

48. Giuggioli D, Manfredi A, Vacchi C, et al. Procedural pain management in the treatment of scleroderma digital ulcers. Clin Exp Rheumatol 2015: 33: 5-10.

49. Ughi N, Crotti C, Ingegnoli F. Effectiveness and safety of oxycodone/naloxone in the managmeent of chronic pain in patients with systemic sclerosis with recurrent digital ulcers: two case reports. Clin Interventions Aging 2016; 11: 307-311.

50. ${ }^{* *}$ Hachulla E, Hatron P-Y, Carpentier P, et al. Efficacy of sildenafil on ischaemic digital ulcer healing in systemic sclerosis: the placebo controlled SEDUCE study. Ann Rheum Dis 2016: 75: 10092015.

This is important the first multicentre randomised controlled trial of PDE5 inhibition in SSc-related digital ulceration. The healing rate in the placebo treated patients was higher than anticipated. Although the primary end-point was not reached, overall the results were in favour of sildenafil. 51. ${ }^{* *}$ Khanna D, Denton CP, Merkel PA, et al. Effect of macitentan on the development of new ischemic digital ulcers in patients with systemic sclerosis. DUAL-1 and DUAL-2 randomized clinical trials. JAMA 2016; 315: 1975-1988.

The DUAL studies are the two largest multicentre studies of SSc-related digital ulceration. Together they highlight the complexities of randomised controlled trials in SSc, including the need for a standardised and reliable definition of digital ulceration.

52. Pulido T, Adzerikho I, Channick RN, et al. Macitentan and morbidity and mortality in pulmonary arterial hypertension. N Eng J Med 2013; 369: 809-818. 
53. Tingey T, Shu J, Smuczek J, Pope J. Meta-analysis of healing and prevention of digital ulcers in systemic sclerosis. Arthritis Care Res 2013; 65; 1460-1471.

54. Korn JH, Mayes M, Matucci-Cerinic M et al. Digital ulcers in systemic sclerosis. Prevention by treatment with bosentan, an oral endothelin receptor antagonist. Arthritis Rheum 2004; 50: 39853993.

55. Matucci-Cerinic M, Denton CP, et al. Bosentan treatment of digital ulcers related to systemic sclerosis: results from the RAPIDS-2 randomised, double-blind, placebo-controlled trial. Ann Rheum Dis 2011;70:32-38.

56. *Agard C, Carpentier PH, Mouthon L, et al. Use of bosentan for digital ulcers related to systemic sclerosis: a real-life retrospective French study of 89 patients treated since specific approval. Scand J Rheumatol 2014;43:398-402.

This study provides insight into the characteristics of patients being prescribed bosentan in France for prevention of new digital ulcers, between 2007 and 2010.

57. De Cata, Inglese M, Molinaro F, et al. Digital ulcers in scleroderma patients: a retrospective observational study. Int J Immunopathol Pharmacol 2016; 29: 180-187.

58. Neumeister MW. The role of botulinum toxin in vasospastic disorders of the hand. Hand Clinics 2015; 31: 23-37.

59. Uppal L, Dhaliwal K, Butler PE. A prospective study of the use of botulinun toxin injections in the treatment of Raynaud's syndrome associated with scleroderma. J Hand Surg 2014; 39E: 876-880.

60. Merritt WH. Role and rationale for extended periarterial sympathectomy in the management of severe Raynaud syndrome: techniques and results. Hand Clinics 2015; 31: 101-120. 
61. Muir M. Surgical management. In: Raynaud's Phenomenon: A Guide to Pathogenesis and Treatment. Edited by FM Wigley, AL Herrick, NA Flavahan (editors). New York: Springer; 2015. pp361-372.

62. *Momeni A, Sorice SC, Valenzuela A, et al. Surgical treatment of systemic sclerosis - is it justified to offer peripheral sympathectomy earlier in the disease process? Microsurgery 2015; 35: 441-446.

This study, albeit with the limits of its retrospective nature, is of interest as it describes outcome in a cohort of patients with SSc undergoing sympathectomy over a 10 year period.

63. Bank J, Fuller SM, Henry GI, Zachary LS. Fat grafting to the hand in patients with Raynaud phenomenon: a novel therapeutic modality. Plast Reconst Surg 2014; 133: 1109.

64. Saito S, Ishii T, Kamogawa Y, et al. Extracorporeal shock wave therapy for digital ulcers of systemic sclerosis: a phase 2 pilot study. Tohoku J Exp Med 2016; 238: 39-47.

65. *Herrick A, Muir L. Raynaud's phenomenon (secondary). Clinical Evidence (Online). BMJ Publishing Group Ltd 2015.

This was a systematic review which aimed to answer the question: 'What are the effects of surgical interventions in complicated secondary Raynaud's phenomenon?' Only two studies met the inclusion criteria, underscoring the need for more research into surgical treatment.

66. Roustit M, Gaillard-Bigot F, Blaise S, et al. Cutaneous iontophoresis of treprostinil in systemic sclerosis: a proof-of-concept study. Clin Pharmacol Ther 2014;95: 439-445.

67. Kotzki S, Roustit M, Arnaud C, et al. Effect of continuous vs pulsed iontophoresis of treprostinil on skin blood flow. Eur J Pharmaceutical Sci 2015; 72: 21-26.

68. Little J, Murray A, Dinsdale G, et al. Whole finger iontophoresis of sodium nitroprusside to increase blood flow in patients with systemic sclerosis: influence of concentration. Int J Pharmaceutics 2015; 490: 446-449. 
69. ${ }^{* *}$ Birgani SA, Mailander M, Wasle I, et al. Efficient therapy of ischaemic lesions with VEGF ${ }_{121^{-}}$ fibrin in an animal model of systemic sclerosis. Ann Rheum Dis (EPub ahead of print).

This paper is potentially very exciting: results from a series of experiments indicated that $\mathrm{VEGF}_{121^{-}}$ fibrin, applied in a gel matrix, induced angiogenesis. This finding should pave the way for studies in humans, the key question being whether this form of treatment could be used to treat SSc-related digital ulcers.

CONFLICTS OF INTEREST

ALH has done consultancy work for Actelion, served on a Data Safety Monitoring Board for Apricus, received research funding and speaker's fees from Actelion, and speaker's fees from GSK. She is or has recently been a principal investigator on studies sponsored by Actelion and Bayer.

Acknowledgements. None

Financial support and sponsorship. None

\section{LEGENDS TO FIGURES}

Figure 1. (a) Fingertip ulcer (ring finger) in a patient with SSc and previous amputations of the index and middle fingers. Mutiple telangiectases can also be seen. (b) An attack of RP (pallor phase).

Figure 2. Modification of the UK Scleroderma Study Group Best Practice Recommendations on the management of Raynaud's phenomenon[34*]. Phosphodiesterase inhibition has been 'moved up' the original pathway to be positioned along with other oral vasodilator therapies. Note that clinicians outside the UK might modify their approach depending on their access to therapies. ACE: 
angiotensin-converting enzyme; ARB: angiotensin receptor blocker: CCB: calcium channel blockers; PDE5: phosphodiesterase type 5; SSRI: selective serotonin reuptake inhibitor.

Figure 3. Modification of the UK Scleroderma Study Group Best Practice Recommendations on the management of SSc-related digital ulceration [34*]. ERA: endothelin-1 receptor antagonist; PDE5: phosphodiesterase type 5 . 
1. Establish diagnosis and identify any underlying cause amenable to treatment

No underlying cause amenable to treatment

Treat underlying cause e.g. cryoglobulinaemia

2. General/lifestyle measures:

Patient education

- Avoid cold, keep warm

- Stop smoking

(Complementary therapies)

Ineffective

3. Drug therapy: first line

CCB, PDE5 inhibitor, ARB, SSRI, alpha

blocker, $A C E_{n}$ inhibitor, topical nitrate

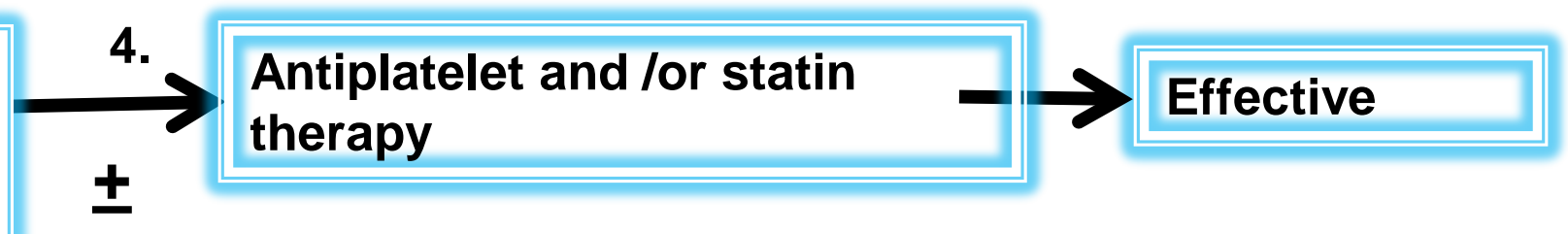

Ineffective oral therapies/refractory disease

5. Drug therapy: refractory

IV prostanoid

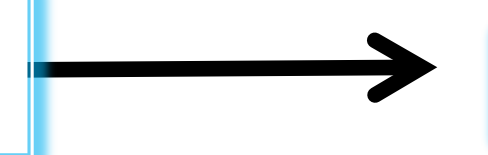

\section{Effective}

Ineffective

6. Progression to digital ulceration and/or critical ischaemia flowchart 
4.

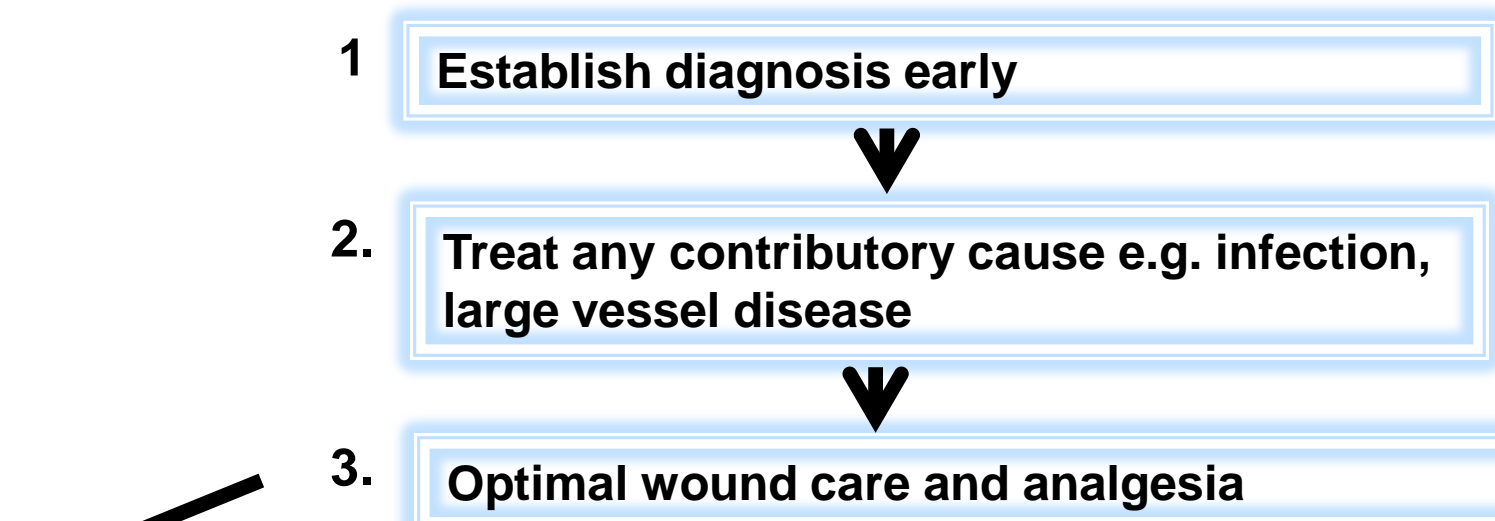

Optimise oral vasodilators (including PDE5 inhibitor) or IV prostanoids
6.

Antiplatelet and/or statin

I therapy

8.

Ineffective/recurrent ulceration

7.

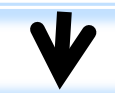

Repeat IV prostanoids or ERA

Ineffective

$\downarrow$

$\checkmark$

Consider digital sympathectomy 